\title{
INVERSE SPECTRAL POSITIVITY FOR SURFACES
}

\author{
PIERRE BÉRARD AND PHILIPPE CASTILLON
}

\begin{abstract}
Let $(M, g)$ be a complete non-compact Riemannian surface. We consider operators of the form $\Delta+a K+W$, where $\Delta$ is the nonnegative Laplacian, $K$ the Gaussian curvature, $W$ a locally integrable function, and $a$ a positive real number. Assuming that the positive part of $W$ is integrable, we address the question "What conclusions on $(M, g)$ and on $W$ can one draw from the fact that the operator $\Delta+a K+W$ is non-negative?"

As a consequence of our main result, we get a new proof of Huber's theorem and Cohn-Vossen's inequality, and we improve earlier results in the particular cases in which $W$ is non-positive and $a=\frac{1}{4}$ or $a \in\left(0, \frac{1}{4}\right)$.
\end{abstract}

MSC(2010): 58J50, 53A30, 53A10.

Keywords: Spectral theory, positivity, minimal surface, constant mean curvature surface.

Published in Rev. Mat. Iberoamericana 30 (2014), 1237-1264.

\section{INTRODUCTION}

Let $(M, g)$ be a complete non-compact Riemannian surface. In the sequel, we will always implicitly assume that $M$ is connected and orientable, without boundary. We denote by $\Delta$ the non-negative Laplacian, by $K$ the Gaussian curvature, and by $\mu$ the Riemannian measure associated with the metric $g$. We denote by $\chi(M)$ the Euler-Poincaré characteristic of $M$, with the convention that $\chi(M)=-\infty$ if $M$ does not have finite topology, see for example [4, Section 1].

In this paper, we consider operators of the form $\Delta+a K+W$, where $a$ is a positive parameter, and $W$ a locally integrable function. Such operators appear naturally when one studies minimal (or constant mean curvature) immersions. Let us mention two examples. The Jacobi (stability) operator of an isometric minimal immersion $M \rightarrow \mathbb{R}^{3}$ into Euclidean 3-space is $\Delta+2 K$. More generally [11, Section 3], the Jacobi operator of a minimal immersion $M \hookrightarrow \widehat{M}^{3}$ into a 3-manifold with scalar curvature $\widehat{S}$ can be written as $\Delta+K-\left(\widehat{S}+\frac{1}{2}|A|^{2}\right)$, where $|A|$ is the norm of the second fundamental form of the immersion.

More precisely, this paper is concerned with the following question: What conclusions on the Riemannian surface $(M, g)$, and on the function $W$, can

Date: November 2014. 
one draw from the fact that the operator $\Delta+a K+W$ is non-negative on $(M, g)$ ? i.e. from the fact that the associated quadratic form is nonnegative on Lipschitz functions with compact support in $M$ (or equivalently on $C^{1}$-functions with compact support),

$$
0 \leq \int_{M}\left(|d f|^{2}+a K f^{2}+W f^{2}\right) d \mu, \quad \forall f \in \operatorname{Lip}_{0}(M) .
$$

Before stating our results, we recall some definitions.

Given a function $W$, we let $W_{+}$and $W_{-}$denote respectively the positive and negative parts of $W, W_{+}=\max \{W, 0\}$ and $W_{-}=\max \{-W, 0\}$, so that $W=W_{+}-W_{-}$and $|W|=W_{+}+W_{-}$.

Definitions. Let $x \in M$, and let $V(r)$ denote the volume of the geodesic ball $B(x, r)$ for the metric $g$. We say that $(M, g)$ has subexponential volume growth if

$$
\limsup _{r \rightarrow \infty} \frac{\ln V(r)}{r}=0 .
$$

We say that $(M, g)$ has polynomial volume growth of degree at most $k$ if

$$
\limsup _{r \rightarrow \infty} \frac{V(r)}{r^{k}}<\infty
$$

We say that $(M, g)$ has $k$-subpolynomial volume growth if

$$
\limsup _{r \rightarrow \infty} \frac{V(r)}{r^{k}}=0
$$

These definitions do not depend on the choice of the point $x \in M$. Note that if $(M, g)$ has polynomial volume growth of degree at most $k$, then it has $k^{\prime}$-subpolynomial volume growth for any $k^{\prime}>k$.

Our main result is the following.

Theorem 1.1. Let $(M, g)$ be a complete non-compact Riemannian surface, and let $W$ be a locally integrable function on $M$, with $W_{+}$integrable. Assume that the operator $\Delta+a K+W$ is non-negative on $M$, and that either

(i) $a \in\left(\frac{1}{4}, \infty\right)$, or

(ii) $a=\frac{1}{4}$, and $(M, g)$ has subexponential volume growth, or

(iii) $a \in\left(0, \frac{1}{4}\right)$, and $(M, g)$ has $k_{a}$-subpolynomial volume growth, with $k_{a}=2+\frac{4 a}{1-4 a}$.

Then,

(A) The surface $(M, g)$ has finite topology, and at most quadratic volume growth. In particular, $(M, g)$ is conformally equivalent to a closed Riemannian surface with finitely many points removed.

(B) The function $W$ is integrable on $(M, g)$, and

$$
0 \leq 2 \pi a \chi(M)+\int_{M} W d \mu .
$$

(C) If $2 \pi a \chi(M)+\int_{M} W d \mu=0$, then $(M, g)$ has subquadratic volume growth, and $a K+W \equiv 0$ a.e. on the surface $M$. 


\section{Remarks.}

(1) Theorem 1.1 does not mention the case in which $M$ is closed. When this is the case, (1.1) implies that,

$$
0 \leq 2 \pi a \chi(M)+\int_{M} W d \mu
$$

with equality if and only if $a K+W \equiv 0$ on $M$. Indeed [11, it suffices to plug the constant function 1 into (1.1), and to notice that equality holds if and only if $\mathbf{1}$ is in the kernel of the operator $\Delta+a K+W$. When $W_{+} \equiv 0$ and $W_{-} \not \equiv 0$, the preceding inequality implies that $(M, g)$ is conformally equivalent to the round sphere; when $W_{+} \equiv 0$ and $\chi(M)=0$, it implies that $W \equiv 0$ and $K \equiv 0$, i.e. that $(M, g)$ is a flat torus.

(2) The volume growth assumptions (ii) and (iii) in the statement of the theorem are sharp, see Section 3.3 for more details.

As a corollary of Assertions A and B in Theorem 1.1, we obtain Huber's theorem and Cohn-Vossen's inequality.

Theorem (Huber's theorem). Let $(M, g)$ be a complete Riemannian surface. Assume that the negative part $K_{-}$of the Gaussian curvature is integrable on M. Then,

(i) $(M, g)$ has finite topology and is conformally equivalent to a closed Riemannian surface with at most finitely many points removed;

(ii) the Gaussian curvature $K$ is integrable, and $\int_{M} K d \mu \leq 2 \pi \chi(M)$ (Cohn-Vossen's inequality).

Proof. Indeed, write $\Delta=\Delta+K+W$ with $W=-K$, so that $W_{+}=K_{-}$, and apply Assertions A and B of Theorem 1.1. Case (i).

The case in which $W_{+} \equiv 0$ is of particular interest. The next two results follow directly from Theorem 1.1 and its proof.

Theorem 1.2. Let $(M, g)$ be a complete non-compact Riemannian surface, and let $q$ be a non-negative locally integrable function on $M$. Assume that the operator $\Delta+a K-q$ is non-negative on $M$, and that either

(i) $a \in\left(\frac{1}{4}, \infty\right)$, or

(ii) $a=\frac{1}{4}$, and $(M, g)$ has subexponential volume growth, or

(iii) $a \in\left(0, \frac{1}{4}\right)$, and $(M, g)$ has $k_{a}$-subpolynomial volume growth, with $k_{a}=2+\frac{4 a}{1-4 a}$.

Then,

(A) The surface $(M, g)$ has at most quadratic volume growth and is conformally equivalent to $\mathbb{C}$ or to $\mathbb{C}^{\bullet}$ with the standard metrics.

(B) The function $q$ is integrable on $(M, g)$, and $\int_{M} q d \mu \leq 2 \pi a \chi(M)$.

(C) If $M$ is a cylinder, then $(M, g)$ has at most linear volume growth and $q \equiv 0$.

Theorem 1.3. Let $(M, g)$ be a complete non-compact 2-dimensional cylinder. Assume that the operator $\Delta+a K$ is non-negative on $(M, g)$, and that either,

(i) $a>\frac{1}{4}$, or 
(ii) $a=\frac{1}{4}$, and $(M, g)$ has subexponential volume growth, or

(iii) $a \in\left(0, \frac{1}{4}\right)$, and $(M, g)$ has $k_{a}$-subpolynomial volume growth, with $k_{a}=2+\frac{4 a}{1-4 a}$.

Then, $(M, g)$ is flat, i.e. $K \equiv 0$.

\section{Remarks.}

(1) Theorem 1.1 and 1.2 extend to the case in which the operator $\Delta+$ $a K+W$ is only assumed to have finite index. The conclusions, under the assumptions of the theorems, are that $(M, g)$ is conformally equivalent to a closed Riemannian surface with a finite number of points removed, and that $W$ is integrable over $(M, g)$. We refer to Section 4 for a precise statement and its proof.

(2) Another interesting situation, with applications to minimal and CMC surfaces, occurs when the potential $q$ has a positive lower bound, i.e. $c=\inf q>0$. Theorem 1.2 can be extended to this situation, improving the results of [7]. We refer to [1] for the details.

Our next result provides an intrinsic version of the optimal length estimate of L. Mazet [16]. Note that this is a local result, we do not need $M$ to be complete. It applies when $M$ is a stable constant mean curvature surface, possibly with boundary, isometrically immersed into a simply connected space form $\widehat{M}$, see Corollary [5.2, Our proof follows the same ideas as in Mazet's paper. We clarify the argument by applying a transplantation method.

Theorem 1.4. Let $(M, g)$ be a Riemannian surface (possibly with boundary $\partial M)$. Assume that the Gaussian curvature satisfies $K \leq \alpha^{2}$ for some $\alpha>0$. Let $J$ be the operator $J=\Delta+a K-c$, with $a \in\left[\frac{1}{2}, \infty\right)$ and $c \geq(a+2) \alpha^{2}$.

(i) If $J$ is non-negative in a geodesic ball $B(x, R)$ contained in $M \backslash \partial M$, then $R \leq \frac{\pi}{2 \alpha}$.

(ii) Assume that the geodesic ball $B\left(x, \frac{\pi}{2 \alpha}\right)$ is contained in $M \backslash \partial M$. If $J$ is non-negative in this ball, then $c=(a+2) \alpha^{2}, K \equiv \alpha^{2}$, and $B\left(x, \frac{\pi}{2 \alpha}\right)$ is covered by the hemisphere $S_{+}^{2}\left(\alpha^{2}\right)$ in the sphere with constant curvature $\alpha^{2}$.

Remark. In the proof of Theorem 1.4, we use the following classical result. Let $\rho:(\widehat{M}, \hat{g}) \rightarrow(M, g)$ be a Riemannian covering. Let $V$ be a locally integrable function on $M$, and let $\hat{V}=V \circ \rho$. According to [11, Theorem 1], $\Delta+V \geq 0$ on $(M, g)$ implies that $\hat{\Delta}+\hat{V} \geq 0$ on $(\widehat{M}, \hat{g})$. It is a natural question to investigate under which conditions the converse statement holds. A partial answer is given by [18, Proposition 2.5]. As a matter of fact, one can show that the converse holds provided that the group $\pi_{1}(\widehat{M})$ is coamenable in the group $\pi_{1}(M)$. We defer the precise statement and its proof to [2] because they rely on techniques and ideas different from those used in the present paper.

\section{Some background for Theorems 1.1, 1.2 and 1.3,}

1. The idea behind the proof of Theorem 1.1 goes back to A. Pogorelov's proof [20] that orientable stable minimal surfaces in $\mathbb{R}^{3}$ are planes. For 
this purpose, he shows that a complete simply-connected surface, with nonpositive curvature and non-negative operator $\Delta+2 K$, must be parabolic. Another proof consists in showing that such a simply-connected surface cannot be conformally equivalent to the unit disk, 3, 11]. In the latter paper, D. Fischer-Colbrie and R. Schoen prove that there exists no complete metric $g$ on the unit disk $\mathbb{D}$, such that $\Delta_{g}+a K_{g} \geq 0$ for some $a \geq 1$. More precisely, they show that the set $I(\mathbb{D}, g)=\left\{a \geq 0 \mid \Delta_{g}+a K_{g} \geq 0\right\}$ is a closed interval which does not contain 1 , and they ask what is the value of the supremum of $I(\mathbb{D}, g)$. This question motivated [4 and the present paper.

2. A. Pogorelov's result was extended to the case in which $\Delta+a K \geq 0$ for some $a>\frac{1}{4}$ by S. Kawai [15]. A more general setting (general topology and curvature, $a>\frac{1}{2}$ ) was considered by R. Gulliver and B. Lawson in [13]. A. Pogorelov's method was improved by T. Colding and W. Minicozzi [5] and, later on, by Ph. Castillon [4] who first proved Case (i) in Theorem 1.2 (with $q \equiv 0$ ); see also [9] and [18] which contain applications to constant mean curvature surfaces in 3-manifolds. Cases (ii) and (iii) in Theorem 1.2 were first considered by J. Espinar and H. Rosenberg in 9], under more restrictive assumptions on $(M, g)$.

3. The main new idea in the proof of Theorem 1.1 is to introduce the function $\widehat{\chi}(t):=\sup \{\chi(B(s)) \mid s \in[t, \infty)\}$, the supremum of the Euler-Poincaré characteristics of open geodesic balls with radius at least $t$, whose jumps describe the large scale topology of $M$, see Section 2. We also introduce new functions to test the positivity of the quadratic form (1.1), see Lemmas 2.4 and 2.5 .

4. Theorem 1.1, Assertion C, and Theorem 1.3 were motivated by the following result due to D. Fischer-Colbrie and R. Schoen [11, Theorem 3] reformulated for our purpose.

Theorem. Let $N$ be a complete oriented 3-manifold of non-negative scalar curvature. Let $M$ be an oriented complete non-compact stable minimal surface in $N$. Then $M$ is conformally equivalent to the complex plane or to a cylinder. If $M$ is a cylinder, and the absolute total curvature of $M$ is finite, then $M$ is flat and totally geodesic.

The proof involves the operator $\Delta+K$. D. Fischer-Colbrie and R. Schoen also point out (Remark 2, p. 207) that "the assumption of finite total curvature should not be essential". This is indeed the case: see 22, proof of Theorem 2], and [19] for a proof using $L^{2}$ harmonic 1-forms. Theorem 1.2 and Theorem [1.3. with $a=1$, provide another method to answer the question raised by D. Fischer-Colbrie and R. Schoen positively.

Remark. Case (i) in Theorem [1.3 appears in [21, Section 3.3] under the assumption that $a \geq 1$; in [9, Theorem 6.3] under the restrictive assumption that $\int_{M} K^{+} d \mu$ is finite; in [8] with a different proof.

The paper is organized as follows. In Section 2, we fix the notations and state some technical lemmas to be used later on. The proof of Theorem 1.1 is given in Section 3.1 (Assertions A and B) and Section 3.2 (Assertion C). The fact that the volume growth assumptions in the theorem are optimal is explained in Section 3.3. In Section 4 we give extensions of Theorems 1.1 
and 1.2 to the case of finite index operators. The proofs of Theorems 1.2 . 1.3 and 1.4 are given in the last section.

The first author was partially supported by CNRS and by the cooperation programmes ARCUS Rhône-Alpes-Brésil and MATH-AMSUD during the preparation of this paper. The authors would like to thank J. Espinar and L. Mazet for their comments on a preliminary version.

\section{Notations AND PRELIMINARY RESUlts}

In this section, we fix some notations which will be used throughout the paper, and we state some preliminary results.

2.1. Notations. In this paper, unless otherwise stated, $(M, g)$ denotes a complete non-compact Riemannian surface without boundary. We also assume that $M$ is connected and orientable.

1. The non-negative Laplacian for the metric $g$ will be denoted by $\Delta$, the Gauss curvature by $K$ and the Riemannian measure by $\mu$.

2. Let $x_{0}$ be a given reference point in $M$. We let $r(x)$ denote the Riemannian distance from the point $x$ to the point $x_{0}$. We let $B(s)$ denote the open geodesic ball with center $x_{0}$ and radius $s$. For $t<s$, we let $C(t, s)$ denote the open set $C(t, s)=B(s) \backslash \bar{B}(t)$. The volume of the ball $B(s)$ is denoted by $V(s)$, the length of the boundary of $B(s)$ by $L(s)$. The length function is a priori only defined for $s \in \mathbb{R}_{+} \backslash E$, where the set of exceptional values $E$ is closed, of Lebesgue measure zero. On the set $\mathbb{R}_{+} \backslash E$, the function $L$ is $C^{1}$ and satisfies Fiala's inequality, see [12],

$$
L^{\prime}(t) \leq 2 \pi \chi(B(t))-\int_{B(t)} K d \mu,
$$

where $\chi(B(t))$ is the Euler-Poincaré characteristic of the ball $B(t)$. As a matter of fact, the function $L$ can be extended to $\mathbb{R}_{+}$. This follows from the work of F. Fiala [12, P. Hartman [14], and K. Shiohama and M. Tanaka [23, 24]. More precisely, there exist two real functions $H, J$ defined on $\mathbb{R}_{+}$, with $H$ absolutely continuous on any compact subset, and $J$ non-decreasing, such that $H(s)-J(s)$ coincides with $L(s)$ when $s$ is not in $E$. The set $E$ and the function $J$ are defined in terms of the cut locus of the point $x_{0}$. The (extended) function $L$ is not continuous in general [14, Figure 1]. However, it satisfies,

$$
L\left(t^{+}\right)=L(t) \text { and } L\left(t^{-}\right) \geq L(t), \quad \forall t>0 .
$$

Furthermore, the function $V$ is differentiable almost everywhere, and $V^{\prime}(s)=$ $L(s)$. From the formula $L=H-J$, one can deduce that

$$
L(b)-L(a) \leq L\left(b^{-}\right)-L(a) \leq \int_{a}^{b} L^{\prime}(t) d t, \text { whenever } 0 \leq a<b .
$$

Remark. In Fiala's paper, $M=\mathbb{R}^{2}$ and $g$ is real analytic. In this case, the set $E$ is discrete. Hartman's paper considers the case $\left(\mathbb{R}^{2}, g\right)$, with $g$ smooth enough. The papers of Shiohama and Tanaka deal with the general case in which $M$ may have finite or infinite topology. All these papers rely on a sharp analysis of the cut locus of a simple closed curve, and on the differential inequality (2.1) satisfied by the length function $L$ away from the 
exceptional set $E$. This was initiated by Fiala, refined by Hartman and later by Shiohama-Tanaka to take into account the transitions from a real analytic to a smooth metric, and from $\mathbb{R}^{2}$ to a general surface $M$.

3. We introduce the total curvature of the ball $B(s)$ to be

$$
G(s)=\int_{B(s)} K(x) d \mu(x) .
$$

4. We denote by $\chi(B(s))$ the Euler-Poincaré characteristic of the open ball $B(s)$. We introduce the function

$$
\widehat{\chi}(s)=\sup \{\chi(B(t)) \mid t \in[s, \infty)\} .
$$

Both functions are continuous to the left. The function $\widehat{\chi}$ is a non-increasing function from $[0, \infty)$ to $\mathbb{Z}$. It has at most countably many discontinuities. We write them as a sequence, finite possibly empty, or infinite tending to infinity,

$$
\left\{t_{j}\right\}_{j=1}^{\bar{N}}=\left\{0<t_{1}<t_{2}<\cdots<t_{n}<\cdots\right\},
$$

with $\bar{N} \in \mathbb{N} \cup\{\infty\}, \bar{N}=0$ when the sequence is empty, and $\bar{N}=\infty$ when it is infinite. Note that this sequence depends on the reference point $x_{0}$.

At the discontinuity $t_{n}, n \geq 1$, the function $\widehat{\chi}$ has a jump

$$
\omega_{n}=\widehat{\chi}\left(t_{n}^{-}\right)-\widehat{\chi}\left(t_{n}^{+}\right), \text {with } \omega_{n} \in \mathbb{N}, \omega_{n} \geq 1 .
$$

Therefore,

$$
\left\{\begin{array}{l}
\widehat{\chi}(s)=1, \text { for } s \in\left[0, t_{1}\right], \text { and } \\
\widehat{\chi}(s)=1-\left(\omega_{1}+\cdots+\omega_{n}\right) \leq-(n-1), \text { for } s \in\left(t_{n}, t_{n+1}\right]
\end{array}\right.
$$

The function $\hat{\chi}$ somehow describes the large scale topology of $M$ as the following lemma shows.

Lemma 2.1. Let $(M, g)$ be a complete Riemannian surface. Let $\left\{t_{j}\right\}_{j=1}^{\bar{N}}$ be the discontinuities of the function $\hat{\chi}$, with jumps $\left\{\omega_{j}\right\}$, relative to some reference point $x_{0}$ in $M$. Let $\chi(M)$ be the Euler-Poincaré characteristic of $M$, with $\chi(M)=-\infty$ if $M$ does not have finite topology. Then

$$
1-\sum_{n=1}^{\bar{N}} \omega_{n} \leq \chi(M)
$$

Proof. Apply [4, Lemma 1.4].

$\diamond$ If $M$ has finite topology, then there exists a value $s_{0}$ such that $\chi(B(s)) \leq$ $\chi(M)$ for all $s \geq s_{0}$. By (2.7), this implies that $1-\sum_{n=1}^{\bar{N}} \omega_{n} \leq \widehat{\chi}\left(s_{0}\right) \leq \chi(M)$. $\diamond$ Otherwise, $\chi(B(s))$ tends to minus infinity when $s$ tends to infinity, so does $\widehat{\chi}(s)$, and formula (2.7) implies that $1-\sum_{n=1}^{\bar{N}} \omega_{n}=-\infty$.

5. As mentioned earlier, the Euler-Poincaré characteristic of balls is related to the length function and to the total curvature of balls. More precisely, 
we have the inequalities,

$$
\left\{\begin{array}{l}
\text { For all } 0 \leq a<b \\
L\left(b^{-}\right)-L(a) \leq 2 \pi(b-a) \sup \{\chi(B(s)) \mid s \in[a, b]\}-\int_{a}^{b} G(s) d s \\
L\left(b^{-}\right)-L(a) \leq 2 \pi(b-a) \widehat{\chi}(a)-\int_{a}^{b} G(s) d s
\end{array}\right.
$$

which follow by integrating the inequality (2.1) satisfied by $L^{\prime}(t)$ for $t \in$ ] $0, \infty \backslash \backslash E[12$, p. 326-328], [14, Proposition 6.1], [23, Proposition 3.7]. Note that we can substitute $L\left(b^{-}\right)$by $L(b)$ in (2.8), because of inequality (2.2).

2.2. Technical lemmas. Definition. Let $0 \leq R<S$. We say that a function $\xi:[R, S] \rightarrow \mathbb{R}$ is admissible in the interval $[R, S]$ if

$$
\left\{\begin{array}{l}
\xi \text { is } C^{1} \text { and piecewise } C^{2} \text { in }[R, S] \\
\xi \geq 0, \xi^{\prime} \leq 0 \text { and } \xi^{\prime \prime} \geq 0
\end{array}\right.
$$

The next two lemmas extend Lemma 1.8 in [4, whose proof uses the method of [5].

Lemma 2.2. For all $0 \leq a<b$, and for all admissible functions $\xi$ on $[a, b]$,

$$
\begin{aligned}
\int_{C(a, b)} K(x) \xi^{2}(r(x)) d \mu(x) \leq & \left.\xi^{2} G\right|_{a} ^{b}-\left.2 \pi \widehat{\chi}(a) \xi^{2}\right|_{a} ^{b}+\left.\left(\xi^{2}\right)^{\prime} L\right|_{a} ^{b^{-}} \\
& -\int_{C(a, b)}\left(\xi^{2}\right)^{\prime \prime}(r(x)) d \mu(x) .
\end{aligned}
$$

Note that in the right-hand side of equation (2.9) one can substitute $\left.\left(\xi^{2}\right)^{\prime} L\right|_{a} ^{b^{-}}$ by $\left.\left(\xi^{2}\right)^{\prime} L\right|_{a} ^{b}$, using (2.2) and the fact that $\xi^{\prime}$ is non-positive.

Proof. We sketch the proof for completeness. First assume that $\xi$ is $C^{2}$. By the co-area formula,

$$
\int_{C(a, b)} K \xi^{2}(r) d \mu=\int_{a}^{b} \xi^{2}(t) G^{\prime}(t) d t
$$

where $G(t)$ is the total curvature of the ball $B(t)$. Introduce the function $H(t):=\int_{a}^{t} G(s) d s$, and integrate the preceding equality by parts twice to get,

$$
\int_{C(a, b)} K \xi^{2}(r) d \mu=\left.\xi^{2} G\right|_{a} ^{b}-\left(\xi^{2}\right)^{\prime}(b) H(b)+\int_{a}^{b} H(t)\left(\xi^{2}\right)^{\prime \prime}(t) d t .
$$

One can estimate $H$ in the right-hand side using (2.8) and the signs of $\xi$ and its derivatives. After some computations and applying the co-area formula once more, one obtains,

$$
\int_{C(a, b)} K \xi^{2}(r) d \mu \leq\left.\left\{\xi^{2} G-2 \pi \widehat{\chi}(a) \xi^{2}+\left(\xi^{2}\right)^{\prime} L\right\}\right|_{a} ^{b^{-}}-\int_{C(a, b)}\left(\xi^{2}\right)^{\prime \prime}(r) d \mu .
$$

This proves the lemma when $\xi$ is $C^{2}$. The fact that the lemma holds for $C^{1}$ and piecewise $C^{2}$ functions $\xi$ follows by cutting the interval into subintervals in which $\xi$ is $C^{2}$. Apply the preceding method in each sub-interval $(c, d) \subset$ $(a, b)$ using an inequality similar to (2.8) with $\widehat{\chi}(a)$ in place of $\widehat{\chi}(c)$; use the 
fact that $\xi$ and $\hat{\chi}$ are non-increasing, and the inequality $L\left(t^{-}\right) \geq L\left(t^{+}\right)=$ $L(t)$ to conclude.

Taking into account the discontinuities $\left\{t_{n}\right\}_{n \geq 1}$ of the function $\hat{\chi}$, see Section 2.1, formula (2.7), we have the following lemma.

Lemma 2.3. Let $\left\{t_{j}\right\}_{j=1}^{\bar{N}}$ be the discontinuities of the function $\hat{\chi}$. Define the index $N(R)$ to be the largest integer $n$ such that $t_{n} \leq R$. Let $t_{0}=0$.

Let $\xi$ be an admissible function in the interval $[R, Q]$. Then,

$$
\begin{aligned}
& \int_{C(R, Q)} K \xi^{2}(r) d \mu \leq 2 \pi\left[\xi^{2}(R) \widehat{\chi}\left(t_{N(R)}\right)-\xi^{2}(Q) \widehat{\chi}\left(t_{N(Q)}\right)\right. \\
& \left.-\sum_{n=N(R)+1}^{N(Q)} \omega_{n} \xi^{2}\left(t_{n}\right)\right]+\left.\xi^{2} G\right|_{R} ^{Q}+\left.\left(\xi^{2}\right)^{\prime} L\right|_{R} ^{Q}-\int_{C(R, Q)}\left(\xi^{2}\right)^{\prime \prime}(r) d \mu .
\end{aligned}
$$

Taking $R=0$ and assuming that $\xi(Q)=0$, we have the inequality

$$
\int_{B(Q)} K \xi^{2}(r) d \mu \leq 2 \pi\left\{\xi^{2}(0)-\sum_{n=1}^{N(Q)} \omega_{n} \xi^{2}\left(t_{n}\right)\right\}-\int_{B(Q)}\left(\xi^{2}\right)^{\prime \prime}(r) d \mu
$$

In particular, assuming that $\xi(Q)=0$, we have the inequality

$$
\int_{B(Q)} K(x) \xi^{2}(r) d \mu \leq 2 \pi \xi^{2}(0)-\int_{B(Q)}\left(\xi^{2}\right)^{\prime \prime}(r) d \mu .
$$

Proof. To prove (2.10), split the integral $\int_{C(R, Q)} K \xi^{2}(r) d \mu$ into a sum,

$$
\int_{C(R, Q)}=\int_{C\left(R, t_{N(R)+1}\right)}+\sum_{n=N(R)+1}^{N(Q)-1} \int_{C\left(t_{n}, t_{n+1}\right)}+\int_{C\left(t_{N(Q)}, Q\right)},
$$

apply Lemma 2.2 and use (2.7). To establish the last two inequalities, use the fact that $\xi(Q)=0$ and $G(0)=L(0)=0$.

The next two lemmas provide admissible functions which we will plug into (1.1) later on.

Lemma 2.4. Fix $0<R<5 R<Q$, and define the function $\xi_{\alpha, \beta, R, Q}$ by

$$
\xi_{\alpha, \beta, R, Q}(t)=\left\{\begin{array}{l}
e^{\left(1-\frac{t}{2 R}\right)^{2}}, \text { for } 0 \leq t \leq R \\
\beta\left(e^{-\alpha t}-e^{-\alpha Q}\right), \text { for } R \leq t \leq Q .
\end{array}\right.
$$

Then, there exists a unique choice $\alpha(R, Q), \beta(R, Q)$ of the parameters $\alpha, \beta$ such that the corresponding function $\xi_{R, Q}$ is admissible in the interval $[0, Q]$. Furthermore,

$$
1 \leq 4 R \alpha(R, Q) \leq 2 \text { and } 1 \leq \beta(R, Q) \leq 10 .
$$

Lemma 2.5. For $a \in\left(0, \frac{1}{4}\right)$, let $\alpha=\frac{2 a}{1-4 a}$ and $\beta=\frac{a}{1-4 a}$. For $0<R<Q$ and $0<\delta$, $\epsilon$, let $\xi_{\delta, \epsilon, R, Q}$ be the function,

$$
\xi(t)=\left\{\begin{array}{l}
\left(1+\frac{t}{R}\right)^{-\beta}, \text { for } t \in[0, R], \\
\delta\left((1+\epsilon t)^{-\alpha}-(1+\epsilon Q)^{-\alpha}\right), \text { for } t \in[R, Q] .
\end{array}\right.
$$


There exists a positive constant $C(\alpha, \beta)>1$, such that for $0<R \leq$ $C(\alpha, \beta) R<Q$, there is a unique choice $\delta(R, Q)$ and $\epsilon(R, Q)$ of the parameters $\delta, \epsilon$, such that the function $\xi_{R, Q}$ defined by equation (2.13) is admissible in the interval $[0, Q]$. Furthermore, there exist positive constants $c_{1}, c_{2}$ such that

$$
1 \leq 6 R \epsilon(R, Q) \leq 2 \text { and } c_{1} \leq \delta(R, Q) \leq c_{2} .
$$

We leave the proofs of these lemmas to the reader.

\section{Proof and optimality of Theorem 1.1}

3.1. Proof of Theorem 1.1-Assertions A and B. In Step 1, we make some preparation. In Step 2, we prove that $M$ has finite topology, and that $W$ is integrable and satisfies $0 \leq 2 \pi a \chi(M)+\int_{M} W d \mu$. We can actually finish the proof of assertions A and B in the Case (i). In Step 3, we prove that $(M, g)$ has at most quadratic volume growth. Steps 2 and 3 both follow from adequate choices of test functions (using Lemma 2.4 and 2.5) in the stability condition (1.1), depending on the case at hand (i), (ii) or (iii).

3.1.1. Step 1. We choose an admissible function $\xi$ on $[0, Q]$, with $\xi(Q)=0$, and we apply the stability condition (1.1) to the Lipschitz function $\xi(r)$, where $r$ is the Riemannian distance to some given point $x_{0} \in M$. We obtain,

$$
\int_{B(Q)} W_{-} \xi^{2}(r) d \mu \leq \int_{B(Q)}\left\{\left(\xi^{\prime}\right)^{2}(r)+a K \xi^{2}(r)\right\} d \mu+\int_{B(Q)} W_{+} \xi^{2}(r) d \mu .
$$

Because $\xi$ is admissible in $[0, Q]$ and $\xi(Q)=0$, we can apply Lemma 2.3, inequality (2.11), and we obtain

$$
\begin{aligned}
& \int_{B(Q)} W_{-} \xi^{2}(r) d \mu+2 \pi a \sum_{n=1}^{N(Q)} \omega_{n} \xi^{2}\left(t_{n}\right) \leq 2 \pi a \xi^{2}(0) \\
& +\int_{B(Q)}\left\{(1-2 a)\left(\xi^{\prime}\right)^{2}(r)-2 a\left(\xi \xi^{\prime \prime}\right)(r)\right\} d \mu+\int_{B(Q)} W_{+} \xi^{2}(r) d \mu,
\end{aligned}
$$

where we have used the notations of Lemma 2.3. Inequality (3.1) holds for all admissible functions $\xi$ in $[0, Q]$ which vanish at $Q$.

Recall (Section 2.1, §4) that the points of discontinuity of the function $\hat{\chi}$ form a sequence $\left\{t_{n}\right\}_{n=1}^{\bar{N}}$ which is either finite possibly empty, or infinite tending to infinity, with stopping index $\bar{N} \in \mathbb{N} \cup\{\infty\}$.

We fix $N$ to be either the stopping index $\bar{N}$, if $\bar{N} \in \mathbb{N}$, or any fixed integer otherwise. We also fix some $R$, with $0<R<Q$. For $Q$ large enough, $Q>t_{N}$ and $Q \geq C(\xi) R$, inequality (3.1) implies that

$$
\begin{aligned}
& \int_{B(R)} W_{-} \xi^{2}(r) d \mu+2 \pi a \sum_{n=1}^{N} \omega_{n} \xi^{2}\left(t_{n}\right) \leq 2 \pi a \xi^{2}(0) \\
& +\int_{B(Q)}\left\{(1-2 a)\left(\xi^{\prime}\right)^{2}(r)-2 a\left(\xi \xi^{\prime \prime}\right)(r)\right\} d \mu+\int_{B(Q)} W_{+} \xi^{2}(r) d \mu,
\end{aligned}
$$

where this inequality holds for any admissible function $\xi$ in $[0, Q]$ vanishing at $Q$, and for any fixed $N$ and $R$ as above. 
The idea is now to apply (3.2) to a function $\xi$ which is well adapted to the case at hand, (i), (ii) or (iii), and to the assertion we want to prove.

3.1.2. Step 2. We will now show that $M$ has finite topology, and that $W$ in integrable over $(M, g)$. We consider the cases (i), (ii) and (iii) separately.

Case (i). Here, $a \in\left(\frac{1}{4}, \infty\right)$. Choose $\xi(t)=\left(1-\frac{t}{Q}\right)^{\alpha}$ for $t \in[0, Q]$, with $\alpha \geq 1$. Then,

$$
(1-2 a)\left(\xi^{\prime}\right)^{2}-2 a \xi \xi^{\prime \prime}=-\frac{\alpha[(4 a-1) \alpha-2 a]}{Q^{2}}\left(1-\frac{t}{Q}\right)^{2 \alpha-2} .
$$

We now fix some $\alpha>\frac{2 a}{4 a-1}$. Plugging the previous equality into inequality (3.2) yields that, for all $R$ and $N$ fixed,

$$
\begin{aligned}
& \int_{B(Q)} W_{-} \xi^{2}(r) d \mu+2 \pi a \sum_{n=1}^{N} \omega_{n} \xi^{2}\left(t_{n}\right) \\
& +\frac{\alpha[(4 a-1) \alpha-2 a]}{Q^{2}} \int_{B(Q)}\left(1-\frac{r}{Q}\right)^{2 \alpha-2} d \mu \leq 2 \pi a+\int_{B(Q)} W_{+} \xi^{2}(r) d \mu .
\end{aligned}
$$

Note that the three terms in the left-hand side of (3.3) are non-negative. Using the fact that $W_{+}$is integrable, we obtain that

$$
\sum_{n=1}^{N} \omega_{n}\left(1-\frac{t_{n}}{Q}\right)^{2 \alpha} \leq 1+\frac{1}{2 \pi a} \int_{M} W_{+} d \mu
$$

Letting $Q$ tend to infinity, we conclude that

$$
\sum_{n=1}^{N} \omega_{n} \leq 1+\frac{1}{2 \pi a} \int_{M} W_{+} d \mu
$$

for any fixed $N$ as above. It follows that $\bar{N}$ is actually finite and, by Lemma 2.1, that $M$ has finite topology.

Remark 3.1. When $W_{+} \equiv 0$, the preceding inequality implies that $\bar{N}=0$, in which case $M$ is homeomorphic to $\mathbb{C}$, or that $\bar{N}=1$ and $\omega_{1}=1$, in which case $M$ is homeomorphic to $\mathbb{C}$ or to $\mathbb{C}^{\bullet}$.

From (3.3) and the previous conclusions, we can choose $N=\bar{N}$ and we obtain,

$$
\left(1-\frac{R}{Q}\right)^{2 \alpha} \int_{B(R)} W_{-} d \mu \leq 2 \pi a\left(1-\sum_{n=1}^{\bar{N}} \omega_{n} \xi^{2}\left(t_{n}\right)\right)+\int_{M} W_{+} d \mu .
$$

Letting $Q$ tend to infinity and using Lemma 2.1, this proves that

$$
\int_{B(R)} W_{-} d \mu \leq 2 \pi a \chi(M)+\int_{M} W_{+} d \mu .
$$

Since this is true for any $R>0$, we have that $W_{-}$is integrable, and Assertion $\mathrm{B}$ follows in the Case (i). 
Note that in Case (i), we can also conclude that $(M, g)$ has at most quadradic volume growth. Indeed, from (3.3), we can infer that there exists a positive constant $C_{\alpha}$ such that

$$
C_{\alpha} Q^{-2} V\left(\frac{Q}{2}\right) \leq 2 \pi a \chi(M)+\int_{M} W_{+} d \mu .
$$

Remark 3.2. When $W_{+} \equiv 0$, we actually get a sharper result when $M$ is homeomorphic to $\mathbb{C}^{\bullet}$ (i.e. to a cylinder). Indeed, in that case $\bar{N}=1$ and $\omega_{1}=1$, and inequality (3.3) gives that

$$
C_{\alpha} Q^{-2} V\left(\frac{Q}{2}\right) \leq 2 \pi a\left\{1-\left(1-\frac{t_{1}}{Q}\right)^{2 \alpha}\right\} .
$$

It follows that $M$ has at most linear volume growth in this particular case.

Note that this completes the proof of Theorem 1.1, Assertions A and B, in the Case (i). In the following arguments, we will concentrate on the cases (ii) and (iii).

Case (ii). Here $a=\frac{1}{4}$ and $(M, g)$ has subexponential volume growth. We choose $\xi(t)=e^{-\alpha t}-e^{-\alpha Q}$ in $[0, Q]$ for some $\alpha>0$. Then,

$$
(1-2 a)\left(\xi^{\prime}\right)^{2}-2 a \xi \xi^{\prime \prime}=\frac{1}{2} \alpha^{2} e^{-\alpha Q} e^{-\alpha t} .
$$

Plugging this equality into (3.2), we obtain, for all $R$ and $N$ fixed,

$$
\begin{aligned}
& \int_{B(R)} W_{-} \xi^{2}(r) d \mu+\frac{\pi}{2} \sum_{n=1}^{N} \omega_{n} \xi^{2}\left(t_{n}\right) \leq \frac{\pi}{2} \xi^{2}(0) \\
& +\frac{1}{2} \alpha^{2} e^{-\alpha Q} \int_{B(Q)} e^{-\alpha r} d \mu+\int_{B(Q)} W_{+} \xi^{2}(r) d \mu .
\end{aligned}
$$

We have the following lemma,

Lemma 3.3. If $(M, g)$ has subexponential volume growth, then for any positive $\alpha$,

$$
\lim _{Q \rightarrow \infty} e^{-\alpha Q} \int_{B(Q)} e^{-\alpha r} d \mu=0 .
$$

Proof. Use the co-area formula and integration by parts.

Let $Q$ tend to infinity in (3.4), and use Lemma 3.3 to obtain,

$$
\int_{B(R)} W_{-} e^{-\alpha r} d \mu+\frac{\pi}{2} \sum_{n=1}^{N} \omega_{n} e^{-2 \alpha t_{n}} \leq \frac{\pi}{2}+\int_{M} W_{+} d \mu
$$

and this inequality holds for all $\alpha>0$ and $N, R$ as above. Letting $\alpha$ tend to zero, we can conclude as in Case (i) that $\bar{N}$ is finite and hence that $M$ has finite topology, and that $W$ is integrable and satisfies,

$$
0 \leq \frac{\pi}{2} \chi(M)+\int_{M} W d \mu .
$$

Remark 3.4. When $W_{+} \equiv 0$, we can conclude as in Remark 3.1 that $M$ is homeomorphic to $\mathbb{C}$ or to $\mathbb{C}^{\bullet}$. 
Note that, unlike in Case (i), we have not yet obtained quadratic volume growth (see Step 3).

Case (iii). Here $a \in\left(0, \frac{1}{4}\right)$ and $(M, g)$ has $k_{a^{-}}$subpolynomial volume growth, with $k_{a}=2+\frac{4 a}{1-4 a}$. We choose $\xi(t)=(1+\epsilon t)^{-\alpha}-(1+\epsilon Q)^{-\alpha}$ in $[0, Q]$, with $\alpha=\frac{2 a}{1-4 a}$ and some $\epsilon>0$. Then,

$$
(1-2 a)\left(\xi^{\prime}\right)^{2}-2 a \xi \xi^{\prime \prime}=2 a \alpha(\alpha+1) \epsilon^{2}(1+\epsilon Q)^{-\alpha}(1+\epsilon t)^{-\alpha-2} .
$$

Plugging this equality into (3.2), we obtain,

$$
\begin{aligned}
& \int_{B(R)} W_{-} \xi^{2}(r) d \mu+2 \pi a \sum_{n=1}^{N} \omega_{n} \xi^{2}\left(t_{n}\right) \leq 2 \pi a \xi^{2}(0) \\
& +2 a \alpha(\alpha+1) \epsilon^{2}(1+\epsilon Q)^{-\alpha} \int_{B(Q)}(1+\epsilon r)^{-\alpha-2} d \mu+\int_{M} W_{+} d \mu .
\end{aligned}
$$

We have the following lemma,

Lemma 3.5. Let $(M, g)$ be a Riemannian surface with $k_{a}$-subpolynomial volume growth, with $k_{a}=2+\frac{4 a}{1-4 a}$. Then, for $\alpha=\frac{2 a}{1-4 a}$ and any $\epsilon>0$,

$$
\lim _{Q \rightarrow \infty}(1+\epsilon Q)^{-\alpha} \int_{B(Q)}(1+\epsilon r)^{-\alpha-2} d \mu=0 .
$$

Proof. Use the co-area formula and integration by parts.

Since both terms in the left-hand side of (3.5) are non-negative, letting $Q$ tend to infinity and using Lemma 3.5, we obtain,

$$
\int_{B(R)} W_{-}(1+\epsilon r)^{-2 \alpha} d \mu+2 \pi a \sum_{n=1}^{N} \omega_{n}\left(1+\epsilon t_{n}\right)^{-2 \alpha} \leq 2 \pi a+\int_{M} W_{+} d \mu,
$$

and this inequality holds for any $\epsilon>0$. Letting $\epsilon$ tend to zero, we obtain,

$$
\int_{B(R)} W_{-} d \mu+2 \pi a \sum_{n=1}^{N} \omega_{n} \leq 2 \pi a+\int_{M} W_{+} d \mu,
$$

and we can conclude as in the previous cases that $\bar{N}$ is finite, that $M$ has finite topology, and that $W$ is integrable, with

$$
0 \leq 2 \pi a \chi(M)+\int_{M} W d \mu .
$$

Remark 3.6. When $W_{+} \equiv 0$, we can show as in Remark 3.1 that $M$ is homeomorphic to $\mathbb{C}$ or to $\mathbb{C}^{\bullet}$.

3.1.3. Step 3. We now show that $(M, g)$ has at most quadratic volume growth. We have already dealt with Case (i) in Step 2. We now consider Cases (ii) and (iii). Recall from Step 2 that $\bar{N}$ is finite.

Case (ii). Here $a=\frac{1}{4}$, and $(M, g)$ has subexponential volume growth. We choose the function $\xi$ to be $\xi_{R, Q}$ as given by Lemma 2.4,

$$
\xi(t)=\left\{\begin{array}{l}
e^{\left(1-\frac{t}{2 R}\right)^{2}}, \text { for } t \in[0, R] \\
\beta\left(e^{-\alpha t}-e^{-\alpha Q}\right), \text { for } t \in[R, Q],
\end{array}\right.
$$


with $0<R<5 Q$ and $\alpha, \beta$ given by the lemma, so that $\xi$ is admissible in $[0, Q]$ and vanishes at $Q$. We apply (3.2) again (making $W_{-} \equiv 0$ which is sufficient for our estimates). For this purpose, we compute,

$$
\left(\xi^{\prime}\right)^{2}-\xi \xi^{\prime \prime}=\left\{\begin{array}{c}
-\frac{1}{2 R^{2}} e^{2\left(1-\frac{t}{2 R}\right)^{2}}, \text { for } t \in[0, R] \\
\alpha^{2} \beta^{2} e^{-\alpha Q} e^{-\alpha t}, \text { for } t \in[R, Q]
\end{array}\right.
$$

and we obtain,

$$
\begin{aligned}
& \frac{1}{4 R^{2}} \int_{B(R)} e^{2\left(1-\frac{r}{2 R}\right)^{2}} d \mu \leq \frac{\pi}{2}\left\{e^{2}-\sum_{n=1}^{\bar{N}} \omega_{n} e^{2\left(1-\frac{t_{n}}{2 R}\right)^{2}}\right\} \\
& +\frac{1}{2} \alpha^{2} \beta^{2} e^{-\alpha Q} \int_{C(R, Q)} e^{-\alpha r} d \mu+\int_{B(Q)} W_{+} \xi^{2}(r) d \mu,
\end{aligned}
$$

where we have chosen $R>t_{\bar{N}}$. We fix $R>t_{\bar{N}}$, and we let $Q$ tend to infinity, using the facts that $\alpha$ and $\beta$ remain controlled, and that the second term in the right-hand side of (3.6) goes to zero when $Q$ tends to infinity because $(M, g)$ has subexponential volume growth (Lemmas 2.4 and 3.3 . Finally, we obtain,

$$
R^{-2} V(R) \leq C\left\{1-\sum_{n=1}^{\bar{N}} \omega_{n} e^{-\frac{t_{n}}{R}\left(2-\frac{t_{n}}{2 R}\right)}\right\}+e^{2} \int_{M} W_{+} d \mu \leq C^{\prime}
$$

for some constant $C^{\prime}$ independent of $R$. This gives that $M$ has at most quadratic volume growth.

Remark 3.7. When $W_{+} \equiv 0$ and $\chi(M)=0$ (which corresponds to $\bar{N}=1$ and $\left.\omega_{1}=1\right)$, the above estimate gives that $M$ has at most linear volume growth.

Case (iii). Here, $a \in\left(0, \frac{1}{4}\right)$ and $(M, g)$ has $k_{a}$-subpolynomial volume growth, with $k_{a}=2+\frac{4 a}{1-4 a}$. We choose the function $\xi$ to be $\xi_{R, Q}$ as given by Lemma 2.5,

$$
\xi(t)=\left\{\begin{array}{l}
\left(1+\frac{t}{R}\right)^{-\beta}, \text { for } t \in[0, R], \beta=\frac{a}{1-4 a}, \\
\delta\left\{(1+\epsilon t)^{-\alpha}-(1+\epsilon Q)^{-\alpha}\right\}, \text { for } t \in[R, Q], \alpha=\frac{2 a}{1-4 a},
\end{array}\right.
$$

with $0<R \ll Q$ and $\delta, \epsilon$ given by the lemma, so that $\xi$ is admissible in $[0, Q]$ and vanishes at $Q$. We apply (3.2) again (making $W_{-} \equiv 0$ which is sufficient for our estimates). For this purpose, we compute,

$$
(1-2 a)\left(\xi^{\prime}\right)^{2}-2 a \xi \xi^{\prime \prime}=\left\{\begin{array}{l}
-\frac{a \beta}{R^{2}}\left(1+\frac{t}{R}\right)^{-2 \beta-2}, \text { for } t \in[0, R], \\
2 a \alpha(\alpha+1) \delta^{2} \epsilon^{2}(1+\epsilon Q)^{-\alpha}(1+\epsilon t)^{-\alpha-2}, \text { for } t \in[R, Q],
\end{array}\right.
$$


and we obtain,

$$
\begin{aligned}
& \frac{a \beta}{R^{2}} \int_{B(R)}\left(1+\frac{r}{R}\right)^{-2 \beta-2} d \mu \leq 2 \pi a\left\{1-\sum_{n=1}^{\bar{N}} \omega_{n}\left(1+\frac{t_{n}}{R}\right)^{-2 \beta}\right\} \\
& +2 a \alpha(\alpha+1) \delta^{2} \epsilon^{2}(1+\epsilon Q)^{-\alpha} \int_{C(R, Q)}(1+\epsilon r)^{-\alpha-2} d \mu+\int_{B(Q)} W_{+} \xi^{2}(r) d \mu
\end{aligned}
$$

where we have chosen $R>t_{\bar{N}}$. We fix $R>t_{\bar{N}}$, and we let $Q$ tend to infinity, using the fact that $\delta$ and $\epsilon$ remain controlled, and that the second term in the right-hand side of (3.7) goes to zero when $Q$ tends to infinity because $(M, g)$ has $k_{a}$-subpolynomial volume growth (Lemmas 2.5] and 3.5). Finally, we obtain,

$$
R^{-2} V(R) \leq C\left\{1-\sum_{n=1}^{\bar{N}} \omega_{n}\left(1+\frac{t_{n}}{R}\right)^{-2 \beta}\right\}+\int_{M} W_{+} d \mu \leq C^{\prime},
$$

for some constant $C^{\prime}$ independent of $R$. It follows that $M$ has at most quadratic volume growth.

Remark 3.8. When $W_{+} \equiv 0$ and $\chi(M)=0$ (which corresponds to $\bar{N}=1$ and $\left.\omega_{1}=1\right)$, the above estimate gives that $M$ has at most linear volume growth.

3.1.4. Conclusion. In the three cases (i), (ii) and (iii), we have proved:

$\diamond$ The surface $M$ has finite topology; when $W_{+} \equiv 0, M$ is homeomorphic to $\mathbb{C}$ or to $\mathbb{C}^{\bullet}$ (Step 2).

$\diamond$ The surface $(M, g)$ has at most quadratic volume growth, and hence [4. Proposition 2.3] is conformally equivalent to a closed Riemannian surface with finitely many points removed (Step 3).

$\diamond$ The function $W$ is integrable and $0 \leq 2 \pi a \chi(M)+\int_{M} W d \mu$; in particular, when $\chi(M)=0$ and $W_{+} \equiv 0$, then $W_{-} \equiv 0$ (Step 2).

$\diamond$ When $W_{+} \equiv 0$ and $\chi(M)=0,(M, g)$ has at most linear volume growth .

The proof of Assertions A and B in Theorem 1.1 is therefore complete.

3.2. Proof of Theorem 1.1 - Assertion C. By Theorem 1.1, Assertions $\mathrm{A}$ and $\mathrm{B}$, we already know that $(M, g)$ has finite topology and, more precisely, that $\bar{N}$ is finite. We also know that $W$ is integrable and satisfies the inequality $0 \leq 2 \pi a \chi(M)+\int_{M} W d \mu$. Assume that

$$
0=2 \pi a \chi(M)+\int_{M} W d \mu
$$

In order to prove Assertion $\mathrm{C}$, we will prove (i) that $(M, g)$ has subquadratic volume growth, and (ii) that $0 \leq a K(x)+W(x)$ for a.e. $x$ in $M$.

3.2.1. Proof that $(M, g)$ has subquadratic volume growth. 
Case $a>\frac{1}{4}$. Taking $\xi(t)=\left(1-\frac{t}{Q}\right)_{+}^{\alpha}$ for some fixed $\alpha$ large enough. Inequality (3.2) becomes

$$
\frac{C_{\alpha}}{Q^{2}} \int_{B(Q)}\left(1-\frac{r}{Q}\right)^{2 \alpha-2} d \mu \leq 2 \pi a\left(1-\sum_{n=1}^{\bar{N}} \omega_{n} \xi^{2}\left(t_{n}\right)\right)+\int_{B(Q)} W \xi^{2}(r) d \mu
$$

The term in the right-hand side can be estimated as follows, using the definition of $\bar{N}$ and the assumption (3.8),

$$
\begin{aligned}
2 \pi a & \left(1-\sum_{n=1}^{\bar{N}} \omega_{n} \xi^{2}\left(t_{n}\right)\right)+\int_{B(Q)} W \xi^{2}(r) d \mu \\
& =2 \pi a\left(1-\sum_{n=1}^{\bar{N}} \omega_{n}\right)+2 \pi a \sum_{1}^{\bar{N}} \frac{2 \alpha t_{n} \omega_{n}}{Q}+o\left(\frac{1}{Q}\right)+\int_{M} W \xi^{2}(r) d \mu \\
& \leq \frac{4 \pi a \alpha}{Q} \sum_{1}^{\bar{N}} t_{n} \omega_{n}+o\left(\frac{1}{Q}\right)+2 \pi a \chi(M)+\int_{M} W \xi^{2}(r) d \mu \\
& \leq \frac{C_{\alpha}^{\prime}}{Q}+o\left(\frac{1}{Q}\right)+\int_{M} W\left(\xi^{2}(r)-1\right) d \mu .
\end{aligned}
$$

It follows that

$$
\frac{C_{\alpha}}{2^{2 \alpha-2}} \frac{V\left(\frac{Q}{2}\right)}{Q^{2}} \leq \frac{C_{\alpha}^{\prime}}{Q}+o\left(\frac{1}{Q}\right)+\int_{M} W\left(\xi^{2}(r)-1\right) d \mu .
$$

Letting $Q$ tend to infinity, and using the dominated convergence theorem in the right-hand side, we conclude that

$$
\lim _{R \rightarrow \infty} \frac{V(R)}{R^{2}}=0
$$

i.e. that $(M, g)$ has subquadratic volume growth.

Case $a=\frac{1}{4}$. We use the same test function as in Section 3.1.3, Case (ii). We choose $R>t_{\bar{N}}$. Inequality (3.2) becomes

$$
\begin{aligned}
& \frac{1}{4 R^{2}} \int_{B(R)} e^{2\left(1-\frac{r}{2 R}\right)^{2}} d \mu \leq \frac{\pi}{2}\left(e^{2}-\sum_{n=1}^{\bar{N}} \omega_{n} e^{2\left(1-\frac{t_{n}}{2 R}\right)^{2}}\right) \\
& +\frac{\alpha^{2} \beta^{2}}{2} e^{-\alpha Q} \int_{C(R, Q)} e^{-\alpha r} d \mu+\int_{B(Q)} W \xi^{2}(r) d \mu .
\end{aligned}
$$


Letting $Q$ tend to infinity, using Lemma 3.3, the definition of $\bar{N}$ and the assumption (3.8), we obtain, for large values of $R$,

$$
\begin{aligned}
\sqrt{e} \frac{V(R)}{4 R^{2}} \leq & \frac{\pi e^{2}}{2}\left(1-\sum_{n=1}^{\bar{N}} \omega_{n} e^{-\frac{t_{n}}{R}\left(2-\frac{t_{n}}{2 R}\right)}\right) \\
& +\int_{B(R)} W e^{2\left(1-\frac{r}{2 R}\right)^{2}} d \mu+\beta^{2} \int_{M \backslash B(R)} W e^{-2 \alpha r} d \mu, \\
\sqrt{e} \frac{V(R)}{4 e^{2} R^{2}} \leq & \frac{\pi}{2}\left(1-\sum_{n=1}^{\bar{N}} \omega_{n}\right)+\frac{\pi}{R} \sum_{n=1}^{\bar{N}} t_{n} \omega_{n}+o\left(\frac{1}{R}\right) \\
& +\int_{B(R)} W e^{-\frac{r}{R}\left(2-\frac{r}{2 R}\right)} d \mu+\frac{\beta^{2}}{e^{2}} \int_{M \backslash B(R)} W e^{-2 \alpha r} d \mu, \\
\sqrt{e} \frac{V(R)}{4 e^{2} R^{2}} \leq & \frac{\pi}{R} \sum_{n=1}^{\bar{N}} t_{n} \omega_{n}+o\left(\frac{1}{R}\right)+\int_{B(R)} W\left(e^{-\frac{r}{R}\left(2-\frac{r}{2 R}\right)}-1\right) \\
& +\frac{\beta^{2}}{e^{2}} \int_{M \backslash B(R)} W e^{-2 \alpha r} d \mu-\int_{M \backslash B(R)} W d \mu .
\end{aligned}
$$

Recall that $\beta$ is uniformy bounded (Lemma 2.4) and that $\alpha>0$. It follows that $|W| e^{-2 \alpha r} \leq|W|$, and that $W$ is integrable (Assertion A). Using the fact that $e^{-\frac{r}{R}\left(2-\frac{r}{2 R}\right)}-1$ tends to zero when $R$ tends to infinity, and the dominated convergence theorem, we can conclude that

$$
\lim _{R \rightarrow \infty} \frac{V(R)}{R^{2}}=0
$$

i.e. that $(M, g)$ has subquadratic volume growth.

Case $0<a<\frac{1}{4}$. We use the same test function as in Section 3.1.3, Case (iii). We choose $R>t_{\bar{N}}$. Inequality (3.2) becomes

$$
\begin{aligned}
& \frac{a \beta}{R^{2}} \int_{B(R)}\left(1+\frac{r}{R}\right)^{-2 \beta-2} d \mu \leq 2 \pi a\left(1-\sum_{n=1}^{\bar{N}} \omega_{n}\left(1+\frac{t_{n}}{R}\right)^{-2 \beta}\right) \\
& +2 a \alpha(\alpha+1) \delta^{2} \epsilon^{2}(1+\epsilon Q)^{-\alpha} \int_{C(R, Q)}(1+\epsilon r)^{-\alpha-2} d \mu \\
& +\int_{B(Q)} W \xi^{2}(r) d \mu .
\end{aligned}
$$


Using Lemma 3.5. for $Q$ tending to infinity and for large values of $R$, we obtain

$$
\begin{aligned}
\frac{a \beta}{2^{2 \beta+2}} \frac{V(R)}{R^{2}} \leq & 2 \pi a\left(1-\sum_{n=1}^{\bar{N}} \omega_{n}\right)+\frac{4 \pi a \beta}{R} \sum_{n} t_{n} \omega_{n}+o\left(\frac{1}{R}\right) \\
& +\int_{B(R)} W\left(1+\frac{r}{R}\right)^{-2 \beta} d \mu+\delta^{2} \int_{M \backslash B(R)} W\left(1+\frac{\epsilon r}{R}\right)^{-2 \alpha} d \mu \\
\frac{a \beta}{2^{2 \beta+2}} \frac{V(R)}{R^{2}} \leq & \frac{4 \pi a \beta}{R} \sum_{n} t_{n} \omega_{n}+o\left(\frac{1}{R}\right)+\int_{B(R)} W\left[\left(1+\frac{r}{R}\right)^{-2 \beta}-1\right] d \mu \\
& -\int_{M \backslash B(R)} W d \mu+\delta^{2} \int_{M \backslash B(R)} W\left(1+\frac{\epsilon r}{R}\right)^{-2 \alpha} d \mu .
\end{aligned}
$$

Recall that $\delta$ is uniformly bounded (Lemma 2.5) and that $|W|\left(1+\frac{\epsilon r}{R}\right)^{-2 \alpha} \leq$ $|W|$ which is integrable. Using the fact that $\left(1+\frac{r}{R}\right)^{-2 \beta}-1$ tends to zero when $R$ tends to infinity, and the dominated convergence theorem, we conclude that

$$
\lim _{R \rightarrow \infty} \frac{V(R)}{R^{2}}=0
$$

i.e. that $(M, g)$ has subquadratic volume growth.

3.2.2. Proof that $a K(x)+W(x) \geq 0$ for a.e. $x \in M$. Recall that,

$$
0 \leq \int_{M}\left(|d f|^{2}+a K f^{2}+W f^{2}\right) d \mu, \quad \forall f \in \operatorname{Lip}_{0}(M) .
$$

Fix some $x \in M$, and take the distance function $r$, the geodesic balls, and the function $\widehat{\chi}(t)$ with respect to this point.

$\diamond$ According to Assertion A, $M$ has finite topology and, more precisely, the function $\widehat{\chi}$ has at most finitely many discontinuities i.e. $\bar{N}$ is finite.

$\diamond$ Let $0<\alpha<1$ and $0<R<t_{1}<t_{\bar{N}}<Q$. Define the function $\xi$ (with parameters $\alpha, R, Q)$ to be

$$
\xi(t)=\left\{\begin{array}{l}
1-\alpha \frac{t}{R}, \text { for } t \in[0, R], \\
(1-\alpha) \frac{Q-t}{Q-R}, \text { for } t \in[R, Q] .
\end{array}\right.
$$

$\diamond$ Use the function $\xi(r)$ to test the positivity condition (3.9). Straightforward computations give

$$
\int_{B(Q)}\left(\xi^{\prime}\right)^{2}(r) d \mu=\frac{\alpha^{2}}{R^{2}} V(R)+\left(\frac{1-\alpha}{Q-R}\right)^{2}(V(Q)-V(R)) .
$$

Applying Lemma 2.3 to the ball $B(R)$ and to the set $C(R, Q)$, another computation yields

$$
\begin{aligned}
& \int_{B(Q)} K \xi^{2}(r) d \mu \leq-\frac{2 \alpha^{2}}{R^{2}} V(R)+2(1-\alpha) \frac{R-\alpha Q}{R(Q-R)} L(R) \\
& +2 \pi\left(1-(1-\alpha)^{2} \sum_{n=1}^{\bar{N}} \omega_{n}\left(\frac{Q-t_{n}}{Q-R}\right)^{2}\right)-2\left(\frac{1-\alpha}{Q-R}\right)^{2}(V(Q)-V(R)) .
\end{aligned}
$$


Finally, we obtain that for $a>0$ and the above choice (3.10) of $\xi$,

$$
\begin{aligned}
& 0 \leq \int_{B(Q)}\left(\left(\xi^{\prime}\right)^{2}(r)+a K \xi^{2}(r)+W \xi^{2}(r)\right) d \mu \leq(1-2 a) \alpha^{2} \frac{V(R)}{R^{2}} \\
& +(1-2 a)\left(\frac{1-\alpha}{Q-R}\right)^{2}(V(Q)-V(R))+2 a(1-\alpha) \frac{R-\alpha Q}{Q-R} \frac{L(R)}{R} \\
& +2 \pi a\left(1-(1-\alpha)^{2} \sum_{n=1}^{\bar{N}} \omega_{n}\left(\frac{Q-t_{n}}{Q-R}\right)^{2}\right) \\
& +\int_{B(R)} W\left(1-\alpha \frac{r}{R}\right)^{2} d \mu+(1-\alpha)^{2} \int_{C(R, Q)} W\left(\frac{Q-r}{Q-R}\right)^{2} d \mu .
\end{aligned}
$$

$\diamond$ The preceding inequality holds for all choices of $\alpha \in(0,1)$ and $0<R<$ $t_{1}<t_{\bar{N}}<Q$. From Section 3.2.1, we know that $(M, g)$ has subquadratic volume growth. Letting $Q$ tend to infinity, we find that

$$
\begin{aligned}
0 \leq & (1-2 a) \alpha^{2} \frac{V(R)}{R^{2}}-2 a \alpha(1-\alpha) \frac{L(R)}{R}+2 \pi a \alpha(2-\alpha) \\
& +2 \pi a(1-\alpha)^{2}\left(1-\sum_{n=1}^{\bar{N}} \omega_{n}\right)+(1-\alpha)^{2} \int_{M} W d \mu \\
& -(1-\alpha)^{2} \int_{B(R)} W d \mu+\int_{B(R)} W\left(1-\alpha \frac{r}{R}\right)^{2} d \mu
\end{aligned}
$$

for all $\alpha \in(0,1)$ and $R \in\left(0, t_{1}\right)$.

$\diamond$ By definition of $\bar{N}$ and using (3.8), we have that,

$$
2 \pi a\left(1-\sum_{n=1}^{\bar{N}} \omega_{n}\right)+\int_{M} W d \mu \leq 0,
$$

and it follows that

$$
\begin{aligned}
0 \leq & (1-2 a) \alpha^{2} \frac{V(R)}{R^{2}}-2 a \alpha(1-\alpha) \frac{L(R)}{R}+2 \pi a \alpha(2-\alpha) \\
& +\int_{B(R)} W\left[\left(1-\alpha \frac{r}{R}\right)^{2}-(1-\alpha)^{2}\right] d \mu .
\end{aligned}
$$

We finally conclude that for all $\alpha \in(0,1)$ and $R \in\left(0, t_{1}\right)$,

$$
\begin{aligned}
0 \leq & (1-2 a) \alpha \frac{V(R)}{R^{2}}-2 a(1-\alpha) \frac{L(R)}{R}+2 \pi a(2-\alpha) \\
& +\int_{B(R)} W\left(1-\frac{r}{R}\right)\left[2-\alpha\left(1+\frac{r}{R}\right)\right] d \mu .
\end{aligned}
$$

$\diamond$ We now use the classical expansions for the length and area of small geodesic balls $B(x, R)$ when $R$ is small,

$$
\begin{aligned}
& L(R)=2 \pi R\left(1-\frac{K(x)}{6} R^{2}+R^{2} \epsilon_{1}(R)\right), \\
& V(R)=\pi R^{2}\left(1-\frac{K(x)}{12} R^{2}+R^{2} \epsilon_{2}(R)\right) .
\end{aligned}
$$


$\diamond$ Plugging (3.13) into (3.12), we find that

$$
\begin{aligned}
0 \leq & \pi \alpha+\frac{K(x) R^{2}}{12}(8 a-(1+6 a) \alpha) \\
& +\pi R^{2}\left((1-2 a) \alpha \epsilon_{2}(R)-4 a(1-\alpha) \epsilon_{1}(R)\right) \\
& +\int_{B(R)} W\left(1-\frac{r}{R}\right)\left[2-\alpha\left(1+\frac{r}{R}\right)\right] d \mu .
\end{aligned}
$$

Letting $\alpha$ tend to zero, we obtain the inequality

$$
0 \leq \frac{2 \pi}{3} a K(x) R^{2}-4 \pi a R^{2} \epsilon_{1}(R)+2 \int_{B(R)} W\left(1-\frac{r}{R}\right) d \mu,
$$

which holds for all $x \in M$ and $R>0$ small enough. To finish the proof, we need to compute the asymptotic expansion of the integral when $R$ tends to zero. By Lebesgue differentiation theorem in local coordinates on the surface $M$ [10, $\S 1.7$, Theorem 1], we have

$$
\int_{B(R)} W d \mu=\pi R^{2} W(x)+o\left(R^{2}\right)
$$

for a.e. $x \in M$ and $R$ small. Moreover, using normal coordinates $(u, r) \in$ $S^{1} \times \mathbb{R}_{+}$centered at $x$, the volume form of $M$ reads $d \mu=\theta_{x}(u, r) d u d r$, where the density function $\theta_{x}$ satisfies $\lim _{r \rightarrow 0} \frac{\theta_{x}(u, r)}{r}=1$, and we have

$$
\begin{aligned}
\frac{1}{R^{3}} \int_{B(R)} r W d \mu & =\frac{1}{R^{3}} \int_{S^{1}} \int_{0}^{R} W(u, r) \frac{\theta_{x}(u, r)}{r} r^{2} d r d u \\
& =\frac{2}{3} \frac{1}{\left(R^{\frac{3}{2}}\right)^{2}} \int_{S^{1}} \int_{0}^{R^{\frac{3}{2}}} W\left(u, s^{\frac{2}{3}}\right) \frac{\theta_{x}\left(u, s^{\frac{2}{3}}\right)}{s^{\frac{2}{3}}} s d s d u .
\end{aligned}
$$

Applying Lebesgue differentiation theorem to the function $W\left(u, s^{\frac{2}{3}}\right) \frac{\theta_{x}\left(u, s^{\frac{2}{3}}\right)}{s^{\frac{2}{3}}}$ on balls of radii $R^{\frac{3}{2}}$ we get

$$
\int_{B(R)} r W d \mu=\frac{2 \pi}{3} R^{3} W(x)+o\left(R^{3}\right)
$$

for a.e. $x \in M$. Finally, for $R$ small, we have

$$
2 \int_{B(R)} W\left(1-\frac{r}{R}\right) d \mu=\frac{2 \pi}{3} R^{2} W(x)+o\left(R^{2}\right)
$$

and inequality (3.14) becomes

$$
0 \leq \frac{2 \pi}{3}(a K(x)+W(x)) R^{2}+o\left(R^{2}\right),
$$

which concludes the proof that $0 \leq a K(x)+W(x)$ for a.e. $x \in M$.

3.2.3. Proof that $a K+W \equiv 0$. Recall from Sections 1 and 3.1 that we already deduced Huber's theorem and Cohn-Vossen's inequality from Theorem 1.1, Assertions A and B.

The first consequence of the inequality $0 \leq a K(x)+W(x)$ for a.e. $x \in M$ is that $a K_{-} \leq W_{+}$a.e. on $M$. Since $W_{+}$is integrable by assumption, it follows that $K_{-}$is integrable and, by Huber's theorem, that $K$ itself is 
integrable. We can then apply Cohn-Vossen's inequality and conclude that $\int_{M} K d \mu \leq 2 \pi \chi(M)$. Finally,

$$
0 \leq \int_{M}(a K+W) d \mu \leq 2 \pi \chi(M)+\int_{M} W d \mu=0 .
$$

It follows that $a K+W \equiv 0$ a.e. on $M$.

3.3. The volume growth assumptions in Theorem 1.1 are optimal. To show that the volume growth assumptions in Theorem 1.1 are optimal, we take $W \equiv 0$.

$\diamond$ The example of the hyperbolic plane shows that Assertion A in Theorem 1.2 cannot hold when $a=\frac{1}{4}$ without an extra assumption on $(M, g)$.

$\diamond$ The examples of the hyperbolic planes of curvature $-c^{2}, c>0$, show that the assumptions in Case (ii) are optimal.

$\diamond$ Consider the unit disk $\mathbb{D}$ with the conformal metric $h_{\alpha}=\left(\frac{2}{1-|z|^{2}}\right)^{2 \alpha}|d z|^{2}$ for $\alpha \geq 1$. The metric $h_{1}$ is the hyperbolic metric with constant curvature -1 . When $\alpha>1$, the metric $h_{\alpha}$ is a complete conformal metric on $\mathbb{D}$, with negative curvature. A simple computation shows that it has polynomial volume growth of degree $2+\frac{1}{\alpha-1}$. Given a complete Riemannian surface $(M, g)$, let $a_{+}(M, g)$ denote the supremum of the numbers $a$ such that $\Delta+a K \geq 0$. This supremum is achieved [4, Proposition 1.1] and $a(\alpha):=a_{+}\left(\mathbb{D}, h_{\alpha}\right)$ is equal to $\frac{1}{4 \alpha}$ by [4, Proposition 4.3]. It follows that the volume growth of $\left(\mathbb{D}, h_{\alpha}\right)$ is polynomial with degree equal to $2+\frac{4 a(\alpha)}{1-4 a(\alpha)}$. This shows that the volume growth assumption in Case (iii) is optimal.

\section{Generalization to finite index operators}

Theorems 1.1 and 1.2 have their counterparts with the assumption that the operator $J$ is non-negative replaced by the assumption that the operator $J$ has finite index. As a matter of fact, one can immediately reduce the former case to the latter by using the following proposition of independent interest.

Proposition 4.1. Let $(M, g)$ be a complete Riemannian manifold, and let $W$ be a locally integrable function on $M$. Then the operator $\Delta+W$ has finite index if and only if there exists a locally integrable function $P$ with compact support such that the operator $\Delta+W+P$ is non-negative.

Proof. Assume that $\Delta+W$ has finite index on $C_{0}^{1}(M)$. Then there exists a compact $K \subset M$ such that $\Delta+W$ is non-negative on $C_{0}^{1}(M \backslash K)$. Take $\phi$ to be a smooth function with compact support, such that $0 \leq \phi \leq 1$ and $\phi \equiv 1$ in a compact neighborhood of $K$. Given any $\psi \in C_{0}^{1}(M)$, write $\psi$ as $\psi=\phi \psi+(1-\phi) \psi$. An easy computation gives,

$$
\begin{aligned}
& \int_{M}|d \psi|^{2}+W \psi^{2}=\int_{M}|d((1-\phi) \psi)|^{2}+W((1-\phi) \psi)^{2} \\
& +\int_{M} W\left(\phi^{2}+2 \phi(1-\phi)\right) \psi^{2}-\frac{1}{2} \int_{M} \psi^{2} \Delta\left((1-\phi)^{2}\right)-\int_{M} \psi^{2}|d \phi|^{2} \\
& +2 \int_{M} \phi\left(1-\frac{1}{2} \phi\right)|d \psi|^{2} .
\end{aligned}
$$


Because $\Delta+W$ is non-negative in $M \backslash K$, and because of our choice of $\phi$, the first and fourth terms in the right-hand side of (4.1) are non-negative. The other terms can be written as $-\int_{M} P \psi^{2}$, where the function $P$ is defined by

$$
P:=|d \phi|^{2}-\Delta\left(\phi\left(1-\frac{1}{2} \phi\right)\right)-W \phi^{2}-2 \phi(1-\phi) W .
$$

Recall that $W$ is locally integrable and that $\phi$ is smooth with compact support. It follows that $P$ is locally integrable, with compact support. By (4.1), the operator $\Delta+W+P$ is non-negative on $C_{0}^{1}(M)$, as stated.

$\diamond$ Assume that there exists a function $P$, which is locally integrable with compact support, such that $\Delta+W+P$ is non-negative on $C_{0}^{1}(M)$. Let $K$ be a compact neighborhood of the support of $P$. Then,

$$
0 \leq \int_{M}|d \psi|^{2}+W \psi^{2}+P \psi^{2}=\int_{M}|d \psi|^{2}+W \psi^{2},
$$

for any $\psi \in C_{0}^{1}(M \backslash K)$, and this means that $\Delta+W$ is non-negative on $C_{0}^{1}(M \backslash K)$. By a result of B. Devyver [6], this implies that $\Delta+W$ has finite index on $C_{0}^{1}(M)$.

Theorem 4.2. Let $(M, g)$ be a complete non-compact Riemannian surface. Let $W$ be a locally integrable function on $M$. Assume that the function $W_{+}$ is integrable on $M$, and that the operator $\Delta+a K+W$ has finite index. Assume furthermore that either,

(i) $a>\frac{1}{4}$, or

(ii) $a=\frac{1}{4}$, and $(M, g)$ has subexponential volume growth, or

(iii) $a \in\left(0, \frac{1}{4}\right)$, and $(M, g)$ has $k_{a}$-subpolynomial volume growth, with $k_{a}=2+\frac{4 a}{1-4 a}$.

Then $W$ is integrable and $(M, g)$ is conformally equivalent to a closed Riemannian surface with finitely many points removed.

Proof. By Proposition 4.1, there exists some function $P$, with compact support, such that $\Delta+a K+W+P$ is non-negative. As $(W+P)_{+}$is still integrable, we can apply Theorem 1.1 to conclude that $(M, g)$ is conformally equivalent to a closed Riemannian surface with finitely many points removed and that $W+P$ is integrable. Since $P$ has compact support, we also have that $W$ is integrable.

\section{OTHER PROOFS}

5.1. Proofs of Theorem 1.2 and $\mathbf{1 . 3}$, To prove Theorem 1.2 and 1.3 , it suffices to follow the proof of Theorem 1.1. Assertions A and B, making $W_{+} \equiv 0, q=W_{-}$, and to use the Remarks 3.1$] 3.6$

5.2. Proof of Theorem 1.4. Let us first consider the case of the sphere with constant curvature $\alpha^{2}, M_{0}=S^{2}\left(\alpha^{2}\right)$. In the sequel, the subscript 0 refers to $M_{0}$. Let $J_{0}=\Delta_{0}+a \alpha^{2}-c$. The operator $J_{0}$ is non-negative in the ball $B_{0}(R)$ if and only if the first Dirichlet eigenvalue of the Laplacian $\Delta_{0}$ in this ball satisfies $\lambda_{1}\left(B_{0}(R)\right) \geq c-a \alpha^{2}$. Since $c \geq(a+2) \alpha^{2}$, it follows that $J_{0}$ non-negative in the ball $B_{0}(R)$ implies that $\lambda_{1}\left(B_{0}(R)\right) \geq \lambda_{1}\left(B_{0}\left(\frac{\pi}{2 \alpha}\right)\right)$, and hence that $R \leq \frac{\pi}{2 \alpha}$, because $\lambda_{1}\left(B_{0}(R)\right)$ is a decreasing function of $R$. If $J_{0} \geq 0$ in $B_{0}\left(\frac{\pi}{2 \alpha}\right)$, then $c=(a+2) \alpha^{2}$, since all previous inequalities become 
equalities. Recall that the first Dirichlet eigenfunction for the Laplacian $\Delta_{0}$ in the hemisphere $B_{0}\left(\frac{\pi}{2 \alpha}\right)$ is $\cos \left(\alpha r_{0}\right)$, up to a scaling factor, where $r_{0}$ is the distance function to a point on the sphere.

Proof of Theorem 1.4. Recall that this theorem is of a local nature. We first state a lemma.

Lemma 5.1. Let $(M, g)$ be a Riemannian surface. Assume that the curvature satisfies $K \leq \alpha^{2}$ for some $\alpha>0$. Let $J$ be the operator $J=\Delta+a K-c$, with $a \in\left[\frac{1}{2}, \infty\right)$ and $c \geq(a+2) \alpha^{2}$. Assume furthermore that the ball $B\left(x, \frac{\pi}{2 \alpha}\right)$ is contained in $M$, for some $x \in M$. Then the least eigenvalue of the operator $J$ with Dirichlet boundary conditions in this ball is non-positive. If $J$ has least Dirichlet eigenvalue 0 in the ball $B\left(x, \frac{\pi}{2 \alpha}\right)$, then $c=(a+2) \alpha^{2}$, $K \equiv \alpha^{2}$ and $B\left(x, \frac{\pi}{2 \alpha}\right)$ is covered by the hemisphere $S_{+}^{2}\left(\alpha^{2}\right)$ in the sphere with constant curvature $\alpha^{2}$.

Clearly, the lemma implies the theorem. Indeed, Assertion (i) follows from the lemma and from the monotonicity of eigenvalues with respect to domain inclusion. Assertion (ii) follows immediately.

Proof of the lemma.

$\diamond$ First observe that we can reduce to the case $a \in\left[\frac{1}{2}, 2\right]$. Indeed, if $a>2$, then for any $a^{\prime} \in\left[\frac{1}{2}, 2\right]$, we can write

$$
\Delta+a K-c=\Delta+a^{\prime} K+\left(a-a^{\prime}\right) K-c \leq \Delta+a^{\prime} K-c^{\prime},
$$

where $c^{\prime}=c+\left(a^{\prime}-a\right) \alpha^{2} \geq\left(a^{\prime}+2\right) \alpha^{2}$. Moreover, if $c^{\prime}=\left(a^{\prime}+2\right) \alpha^{2}$, then $c=(a+2) \alpha^{2}$.

$\diamond$ Assume that $a \in\left[\frac{1}{2}, 2\right]$. Let $A:=\frac{\pi}{2 \alpha}$. Because $K \leq \alpha^{2}$, the map $\exp _{x}$ : $T_{x} M \rightarrow M$ is a local diffeomorphism on the ball $D(0, A)$. Let $\tilde{g}=\exp _{x}^{*} g$ be the pulled-back metric to $T_{x} M$. Let $\mu_{1}$ be the least Dirichlet eigenvalue of $\Delta+a K-c$ in $B(x, A)$. Then, $\Delta+a K-c-\mu_{1} \geq 0$ in $B(x, A)$ and hence, there exists a positive function $u: B(x, A) \rightarrow \mathbb{R}$ such that $\left(\Delta+a K-c-\mu_{1}\right) u=0$, see [11]. Let $\tilde{u}=u \circ \exp _{x}$. Because $\exp _{x}$ is a local isometry, we have $\left(\tilde{\Delta}+a \tilde{K}-c-\mu_{1}\right) \tilde{u}=0$ and hence the least Dirichlet eigenvalue $\tilde{\mu}_{1}$ of $\tilde{\Delta}+a \tilde{K}-c$ satisfies $\tilde{\mu}_{1} \geq \mu_{1}$. To show that $\mu_{1}$ is non-positive, it suffice to show that $\tilde{\mu}_{1}$ is non-positive. We have reduced to the simply-connected case.

$\diamond$ We now work in the simply-connected disk $D(0, A)$, with a metric (also denoted) $g$ such that $K \leq \alpha^{2}$. We denote by $L(r)$ the length of $\partial D(0, r)$ for this metric and we let $L_{0}(r)$ be the corresponding length on the sphere, $L_{0}(r)=2 \pi \frac{\sin (\alpha r)}{\alpha}$. By Bishop's comparison theorem, we have that

$$
L(r) \geq L_{0}(r) .
$$

We now use Pogorelov's trick. Let $\xi:[0, A] \rightarrow \mathbb{R}$ be a $C^{2}$ function such that $\xi(0)=1$ and $\xi(A)=0$. We compute the quadratic form $\mathcal{Q}$ associated with $J=\Delta+a K-c$ on the function $\xi(r)$, where $r$ is the geodesic distance to 0 in $D(0, A)$. We also introduce the total curvature of $D(0, r)$,

$$
G(r)=\int_{D(r)} K d \mu
$$


with respect to the Riemannian measure in $D(0, A)$. Applying the co-area formula,

$$
\begin{aligned}
\mathcal{Q}(\xi(r)) & =\int_{D(0, A)}\left(|d \xi(r)|^{2}+(a K-c) \xi^{2}(r)\right) d \mu \\
& =\int_{0}^{A}\left(\left(\xi^{\prime}\right)^{2}-c \xi^{2}\right)(t) L(t) d t+a \int_{0}^{A} G^{\prime}(t) \xi^{2}(t) d t,
\end{aligned}
$$

and we compute the second integral in the right-hand side by integration by parts,

$$
\mathcal{Q}(\xi(r))=\int_{0}^{A}\left(\left(\xi^{\prime}\right)^{2}-c \xi^{2}\right)(t) L(t) d t-a \int_{0}^{A} G(t)\left(\xi^{2}\right)^{\prime}(t) d t
$$

By the Gauss-Bonnet formula, we have $G(t)=2 \pi-L^{\prime}(t)$ so that (5.3) becomes, after another integration by parts,

$$
\mathcal{Q}(\xi(r))=\int_{0}^{A}\left(\left(\xi^{\prime}\right)^{2}-c \xi^{2}-a\left(\xi^{2}\right)^{\prime \prime}\right)(t) L(t) d t+2 \pi a .
$$

Finally, we obtain

$$
\mathcal{Q}(\xi(r))=\int_{0}^{A}\left((1-2 a)\left(\xi^{\prime}\right)^{2}-2 a \xi \xi^{\prime \prime}-c \xi^{2}\right)(t) L(t) d t+2 \pi a,
$$

for any function $\xi:[0, A] \rightarrow \mathbb{R}$ which is $C^{2}$ and such that $\xi(0)=1$ and $\xi(A)=0$.

We now use the test function $\eta(r)=\cos (\alpha r)$ in formula (5.4), where $r$ is the Riemannian distance to the center of the ball, i.e. we transplant the first eigenfunction of the hemisphere to a function on the ball $D(0, A)$.

$$
\mathcal{Q}(\eta(r))=\int_{0}^{A}\left((1-2 a) \alpha^{2} \sin ^{2}(\alpha t)+\left(2 a \alpha^{2}-c\right) \cos ^{2}(\alpha t)\right) L(t) d t+2 \pi a,
$$

and hence

$$
\mathcal{Q}(\eta(r)) \leq \int_{0}^{A}\left((1-2 a) \alpha^{2} \sin ^{2}(\alpha t)+(a-2) \alpha^{2} \cos ^{2}(\alpha t)\right) L(t) d t+2 \pi a
$$

where we have used the fact that $c \geq(2+a) \alpha^{2}$. Recall that $a \in\left[\frac{1}{2}, 2\right]$. Using the inequality (5.1), we find that

$$
\mathcal{Q}(\eta(r)) \leq 2 \pi a+\int_{0}^{A}\left((1-2 a) \alpha^{2} \sin ^{2}(\alpha t)+(a-2) \alpha^{2} \cos ^{2}(\alpha t)\right) L_{0}(t) d t .
$$

The right-hand side of (5.6) is zero because this is the value of the quadratic form of the operator $J_{0}=\Delta-2 \alpha^{2}$ on the hemisphere $S_{+}^{2}\left(\alpha^{2}\right)=B_{0}\left(\frac{\pi}{2 \alpha}\right)$. We conclude that $\mathcal{Q}(\eta(r)) \leq 0$ and hence that the least Dirichlet eigenvalue of $\Delta+a K-c$ in $D(0, A)$ is non-positive, as stated in the lemma. If this eigenvalue is zero, then $\mathcal{Q}(\eta(r))=0$, and we must have equality in both (5.5) and (5.6), i.e. $c=(2+a) \alpha^{2}$ and $L(t) \equiv 2 \pi \frac{\sin (\alpha t)}{\alpha}$. We then deduce that $G(t) \equiv 2 \pi(1-\cos (\alpha t))$. Since $K \leq \alpha^{2}$, integrating $K$ we find that $K \equiv \alpha^{2}$ and hence we conclude that $D(0, A)=S_{+}^{2}\left(\alpha^{2}\right)$. This proves the lemma.

As a corollary of Theorem 1.4, we obtain Mazet's estimates. 
Corollary 5.2. Let $(M, g) \rightarrow(\widehat{M}, \hat{g})$ be an isometric immersion with constant mean curvature $H$ in a simply connected space form with constant sectional curvature $\kappa$. Assume furthermore that $H^{2}+\kappa>0$ and that the immersion is (strongly) stable. Then,

$$
d_{g}(x, \partial M) \leq \frac{\pi}{2 \sqrt{H^{2}+\kappa}},
$$

where $d_{g}(x, \partial M)$ is the distance from $x \in M$ with respect to the metric $g$ to the boundary of $M$, with equality if and only if $M$ is the hemisphere of a sphere of mean curvature $H$ in $\widehat{M}$.

Proof. The Jacobi operator of the immersion is $J=\Delta-|A|^{2}-\widehat{\operatorname{Ric}}(n)$, where $A$ is the second fundamental form of the immersion and $n$ the unit normal along the immersion. By the Gauss equation, we find that $J=\Delta+$ $2 K-4\left(H^{2}+\kappa\right)$ and that $K=H^{2}+\kappa-\frac{1}{4}\left(k_{1}-k_{2}\right)^{2}$, where $k_{i}$ are the principal curvatures. We can apply Theorem 1.4 with $a=2$ and $\alpha^{2}=H^{2}+\kappa$. For the equality case, note that equality implies that $M$ is totally umbilic.

\section{Remarks.}

(1) This corollary provides a unified proof of Theorem 3.1 and Corollary 3.2 in Mazet's paper [16, without using Lawson's correspondence.

(2) The proof of Theorem 1.4 is simpler than that of [16, Theorem 3.1], but it uses the same idea which goes back to A. Pogorelov [20].

(3) It is not clear whether the assumptions $a \geq \frac{1}{2}$ and $c \geq(a+2) \alpha^{2}$ are sharp.

\section{REFERENCES}

[1] Bérard, P. and Castillon, P. Remarks on J. Espinar's "Finite index operators on surfaces" arXiv:1204.1604v1

[2] Bérard, P. and Castillon, P. Spectral positivity and Riemannian coverings Bull. London Math. Soc. 45 (2013), no. 5, 1041-1048

[3] Carmo do, M. and Peng, C-K. Stable complete minimal surfaces in $\mathbb{R}^{3}$ are planes Bull. Amer. Math. Soc. 1 (1979), no. 6, 903-906

[4] Castillon, P. An inverse spectral problem on surfaces Comment. Math. Helv. 81 (2006), 271-286

[5] Colding, T. and Minicozzi, W. Estimates for parametric elliptic integrands Internat. Math. Res. Notices 6 (2002), 291-297

[6] Devyver, B. On the finiteness of the Morse index for Schrödinger operators Manuscripta Math. 139 (2012), no. 1-2, 249-271

[7] Espinar, J.M. Finite index operators on surfaces J. Geometric Analysis 23 (2013), no. 1, 415-437

[8] Espinar, J.M. Rigidity of stable cylinders in three-manifolds Proc. Amer. Math. Soc. 140 (2012), no. 5, 1769-1775

[9] Espinar, J. and Rosenberg, H. A Colding-Minicozzi inequality and its applications Trans. Amer. Math. Soc. 363 (2011), no. 5, 2447-2465

[10] Evans, L.C. and Gariepy, R.F. Measure theory and fine properties of functions CRC Press, Boca Raton, FL, 1992

[11] Fischer-Colbrie, D. and Schoen, R. The structure of complete stable minimal surfaces in 3-manifolds of non-negative scalar curvature Comm. Pure Applied Math. 33 (1980), 199-211

[12] Fiala, F. Le problème des isopérimètres sur les surfaces ouvertes à courbure positive Comment. Math. Helv. 13 (1940-1941), 293-346 
[13] Gulliver, R. and Lawson, H.B. The structure of minimal hypersurfaces near a singularity Proc. Symp. Pure Math. 44 (1986), 213-237

[14] Hartman, P. Geodesic parallel coordinates in the large Amer. J. Math. 86 (1964), no. $4,705-727$

[15] Kawai, S. Operator $\Delta-a K$ on surfaces Hokkaido Math. J. 17 (1988), 147-150

[16] Mazet, L. Optimal length estimates for stable CMC surfaces in 3-space forms Proc. Amer. Math. Soc. 137 (2009), no. 8, 2761-2765

[17] Meeks, W.H., Pérez, J. and Ros, A. Liouville-type properties for embedded minimal surfaces Comm. Analysis and Geometry 4 (2006), no. 4, 703-723

[18] Meeks, W.H., Pérez, J. and Ros, A. Stable constant mean curvature surfaces In Handbook of Geometric Analysis, Vol. 1. Lizhen Ji, Peter Li, Richard Schoen and Leon Simon Editors. International Press, 2008

[19] Miyaoka, R. $L^{2}$ harmonic 1-forms on a complete stable minimal hypersurface In Geometry and global analysis Ed. T. Kotake, S. Nishikawa, R. Schoen. Sendai 1993, pp. 289-293

[20] Pogorelov, A.V. On the stability of minimal surfaces Soviet Math. Dokl. 24 (1981), $274-276$

[21] Reiris, M. Geometric relations of stable minimal surfaces and applications arXiv: $1002.3274 \mathrm{v} 1$

[22] Schoen, R. and Yau, S.T. Complete three dimensional manifolds with positive Ricci curvature and scalar curvature In Seminar on differential geometry Ed. S.T. Yau. Annals of Math. Studies 102 (1982), 209-228

[23] Shiohama, K. and Tanaka, M. An isoperimetric problem for infinitely connected complete open surfaces In Geometry of Manifolds (Mastumoto, 1988), Prospect. Math. 8. Academic Press 1989, 317-343

[24] Shiohama, K. and Tanaka, M. The length function of geodesic parallel circles In Progress in differential geometry, (K. Shiohama Ed.). Adv. Stud. Pure Math. 22, Math. Soc. Japan, Tokyo 1993, 299-308

Pierre Bérard

Université de Grenoble

Institut Fourier (UJF-CNRS)

B.P. 74

38402 Saint Martin d'Hères Cedex

France

pierrehberard@gmail.com
Philippe Castillon

Université Montpellier II

Dépt des sciences mathématiques CC 51

I3M (UMR 5149)

34095 Montpellier Cedex 5

France

philippe.castillon@univ-montp2.fr 